\title{
Minimizing radiation injury and neoplastic effects during pediatric fluoroscopy: what should we know?
}

Published online: 22 July 2006

(C) Springer-Verlag 2006
What we know from the history of fluoroscopically guided intervention in children and in adults is that radiation injury is very rare and that there are certain factors that increase the risk or exacerbate the severity of radiation-induced injury [1-3], namely:

1. Injury is most likely to occur when the X-rays must pass through a thick amount of tissue, e.g., in large patients or when steep oblique or cranial/caudal angulation is employed.

2. Injury almost always results from long procedures that involve a fixed orientation over the same skin entrance site.

3. Sometimes extraneous body parts are involved, and this enhances the risk for injury.

4. Physicians are often unaware that fluoroscopic radiation can lead to radiation injury, resulting in lax radiation management practices.

Effects of radiation in both the pediatric patient and the pediatric fluoroscopist are a realistic possibility that must be addressed by diligent radiation management. Some frontline aspects of that management are addressed in this article.

\section{Experience with injury in children}

Perhaps the concept of radiation injury from fluoroscopically guided pediatric intervention seems incongruous with the concept that dose rates are much lower in children since they are much smaller than adults. However, not all children are small, and there are some special concerns that warrant attention.

\section{K. Wagner $(\square)$}

Department of Diagnostic and Interventional Imaging,

The University of Texas Medical School at Houston,

6431 Fannin St.,

Houston, TX 77030, USA

e-mail: Louis.K.Wagner@uth.tmc.edu

Tel.: +1-713-5007671

Fax: $+1-713-5007676$

\section{Experience with injury in physicians}

That contemporary physicians have been injured by inappropriate use of radiation is also a fact in the history books $[4,5]$. The circumstances surrounding these events are important lessons. Injuries include dermatitis, sometimes severe, on the hands and arms of interventionalists, radiation-induced cataract, and hair loss on the legs.

\section{Cancer in patients and in practitioners}

It is known that children and fetuses are at risk of cancer from medical radiation. This is reviewed in other articles of this journal and need not be repeated here. Of greatest concern are risks of leukemia, female breast cancer, thyroid 
cancer, lung cancer and brain cancer. Good radiation management is necessary to limit these risks.

\section{Deterministic versus stochastic effects}

A deterministic biological effect, such as radiation skin injury, is one that involves many cells before it can be expressed. Therefore, a certain minimum amount of radiation, called the threshold dose, will have to be delivered before the effect can occur. When the threshold is surpassed, the likelihood of inducing the effect increases rapidly, and the severity of the effect increases as dose continues to increase. This is in contrast to a stochastic effect, such as induced cancer, where interaction in a single cell has the possibility of inducing the effect, implying that there exists no threshold dose and only the probability of induction increases with dose, not the severity.

The lesson to be learned is that deterministic and stochastic effects in children and in practitioners are possible, and certain measures can be taken to limit their likelihood or to eliminate them. It is important that we recognize injuries and properly advise patients on what to do should they occur. A discussion of the mechanisms of injury and the threshold doses for their occurrence can be found in Koenig et al. [2].

\section{The FDA Advisory}

In 1994, the United States Food and Drug Administration issued the only advisory that it has ever issued on medical radiation effects [6]. In that advisory the FDA warned physicians and health-care administrators that the FDA had received reports of occasional but severe skin injuries in patients undergoing certain fluoroscopically guided procedures. The FDA delineated those procedures likely to lead to high skin doses and provided some recommendations on how to reduce the likelihood of injury. Topics of these recommendations included:

1. Training of physicians

2. Dose management

3. Dose monitoring and recording

4. Patient counseling

Although these recommendations are not regulatory, they do represent advice from one of the highest health authorities in the United States. Familiarization with the FDA Advisory is recommended to ensure that practices at a particular facility have appropriately addressed these recommendations. Organizations that have developed documents addressing this Advisory, at least in part, include the Society of Interventional Radiology [7] and the American College of Cardiology Foundation along with its sister societies [8]. The American College of Radiology has for years recommended that training of radiologists include radiation management.

\section{Lessons from radiation effects in children}

Skin injury in arms

Vañó et al. [1] have reported on radiation-induced skin injury in the right arms of two pediatric cardiology patients. One was a 7-year-old girl and the other a 12-year-old girl. Both underwent prolonged electrophysiological and ablation procedures. During the procedures, the patients' right arms were in the lateral beam as the patient reclined on the table. This geometry causes several important problems. First, the added tissue in the field-of-view causes unnecessary attenuation of the X-rays. This in turn causes the automatic brightness control of the fluoroscope to raise the radiation output in compensation for the added attenuation. Second, the closer proximity of the arm to the X-ray port means that the intensity of the beam at the surface of the arm is exponentially enhanced according to the inverse square law. Finally, the added tissue likely causes the $\mathrm{kVp}$ to increase, resulting in reduced contrast, perhaps prolonging the procedure.

Although it is important to rest the arms in a comfortable position so as not to compromise circulation, it is also important to position them out of the way of the beam in order to avoid radiation risk to the arm. In adults, there exist several instances of severe radiation injuries to arms, all caused during prolonged fluoroscopically guided interventional procedures $[2,3,8,9]$.

The rule to remove arms from the beam applies to both arms. The presence of the arm on the exit-beam side of the patient also serves to attenuate radiation, enhancing radiation output and reducing image contrast.

\section{Chest and breast}

Vañó et al. [1] have also reported on a 17-year-old girl who underwent two attempts at a cardiac ablation procedure. This resulted in a large area of skin scarring on the right side of her chest from a left lateral projection, and the scar bordered on the posterior aspects of her right breast. The scarring included hyper- and hypopigmentation, telangiectasia and induration. The patient had limited movement of her right arm.

\section{Summary of effects in children}

There are two lessons from these experiences. The first is that prolonged and repeated procedures during which the same skin area is irradiated and which involve oblique, lateral, or similar beam projections place the skin at potential risk. The second lesson involves a more subtle concern of radiation-induced cancer. The case of the 17year-old girl demonstrates that large doses are superficially delivered by the entrance beam. If the female breast is placed in direct view of or in proximity to the entrance beam, large doses of radiation may be delivered to this radiosensitive organ during a stage in life when it is most 
vulnerable (children being more sensitive to the carcinogenic effects of X-rays than adults). This teaches us that efforts should also be made to avoid, to the extent possible, the direct and indirect exposure of female breast tissue. This goal can be achieved either by strategically planning beam orientations that distance the beam from the breast or by the appropriate application of collimation to limit direct and scattered radiation to the breast.

Head, hair, and eyes

Interventional procedures in the head periodically result in hair loss. Hair loss in pediatric patients has been documented. Although hair loss is primarily a cosmetic concern, it does signal that certain threshold doses of radiation have been crossed and it teaches us to be cautious about other potential effects.

The frontal view of fluoroscopy during an interventional procedure is routinely delivered with the X-ray beam entering posteriorly and exiting anteriorly. This is traditional and is done this way for the safety of the operator and the child. The threshold dose for hair loss is about $3 \mathrm{~Gy}$. The threshold for induced cataract is $2 \mathrm{~Gy}$, or possibly less. Thus, had the same beam been delivered anterior-posterior through the eyes first, the probability of inducing cataract would have been substantial.

This fact raises a consideration for the eyes from the lateral beam. Sometimes the orbits are in the field-of-view. Consideration should always be given to using the collimators to cone the beam to an area that excludes as much of the orbit as possible. This will substantially limit the dose to the lens. In all procedures involving potential direct exposure of the orbits, counseling the patient's guardian regarding the risk of radiation-induced cataract should be considered.

Further uses of collimation to reduce risk

The thyroid of a child is known to be particularly sensitive to the carcinogenic nature of radiation. Collimation during any procedure in the head or chest limits the potential risk to this organ as well as to all organs in the body. Collimation is of particular importance in all pediatric interventions because of the smaller size of the child and the large percentage of organs that can be exposed to the beam. Counseling the patient's guardian on the risks of cancer later in life might be an important consideration.

When counseling a patient's guardian on cancer risks, the author prefers to quote the risk as the likelihood the child will not develop cancer and compare this to the normal risk. For example, the risk of a child developing cancer later in life from an interventional cardiac procedure has been estimated as about $1 \%$ in some cases (this depends on a lot of assumptions and does not apply in all cases). The patient's guardian is told that the chances are better than $99 \%$ that the child will have the same chance of a healthy life as any other child, within the framework of children with a similar medical condition. Placing risk in the positive frame provides a lot better perspective than trying to deal with the negative perspective of abstract absolute risks.

\section{Bone}

Although the author knows of no reported effects of fluoroscopic radiation on growing bone in children, growth arrest is a potential consideration [10]. This concern is highest in superficial bone associated with the face, skull, and ribs. Soft tissue neighboring calcified bone receives an amplified dose over that of soft tissue alone. This is because of the photoelectric interaction in calcium. The ejected electron is propelled a few microns into the soft tissue, and this amplifies the dose effect. Of course, in poorly calcified bone or in cartilage, this amplification effect is less or nonexistent. Detectable growth stunting has been observed in rats at absorbed doses of $6 \mathrm{~Gy}$. It is reasonable to state, based on previous reports, that effects in bone are not likely to be observed unless doses are sufficiently high to induce serious skin reactions.

\section{Radiation protection for and injuries in contemporary fluoroscopists}

Hands

Radiation exposure to the hands of a fluoroscopist during pediatric fluoroscopy is sometimes necessary, particularly in small children. Here are some key protection features that will help in managing risk to the operator:

1. Keep hands and fingers out of the beam unless necessary for the safety of the patient

2. Work on the exit beam side of the patient to avoid the higher dose rates to the hands from the entrance beam

3. For lateral beams, rotate the collimators to be parallel to the patient surface and collimate down to below the patient surface, thus eliminating the hands and fingers from direct exposure

4. Use remote handling devices, such as forceps, when safe to do so

5. Wear a radiation ring monitor and review your hand dose periodically

Radiation ring monitors must be used effectively. This requires that they be worn on the hand likely to get more exposure; that they be worn on the finger likely to get the highest dose; that they be worn with the sensitive element facing the oncoming beam to record the entrance finger dose, not the exit dose; and that they be worn on the middle phalanx if workable to get the monitor as close as possible to the most at-risk anatomy. The physician's comfort is essential to the safe completion of the procedure, and these are ideal recommendations. Some compromises might be necessary for the sake of patient care. 
Surgical leaded gloves are sometimes used. Some considerations on the use of these gloves are appropriate. First, the gloves are designed to attenuate X-rays. Therefore, if they are inserted into the beam over the sensor area used by the fluoroscope to drive the automatic exposure rate control, the gloves will be counterproductive. This is because the machine will drive the radiation output higher to penetrate the gloves, nullifying any protective effect and resulting in a higher dose rate to the patient. If placed at the edge of the field-of-view or outside it, the gloves might protect against scatter radiation. However, the protection usually quoted by manufacturers might apply only to a single layer protection from an oncoming beam and not to a hand inside the glove, which might receive more radiation. This is because some of the radiation that penetrates through the finger is backscattered by the glove back into the hand [11]. The gloves are often expensive and some have suggested they produce a false sense of security that might lead physicians into thinking they can expose their hands more frequently because they are "protected" [12]. Protective surgical gloves are an adjunct to applied protection principles, not a substitute for them.

Head and neck

The first and foremost rule to protection of the head and neck area of the fluoroscopist is to wear a radiation monitor at the collar level outside the lead protection on the side of the torso receiving the highest scatter radiation and to review the results periodically. Acceptably safe practices are in place as long as the doses are well within regulatory limits.

Traditionally, the thyroid has been thought to be a very sensitive organ for radiation-induced cancer in fluoroscopists. Data in adults from medical diagnosis with I-131 [13] and from the experience of the Chernobyl nuclear reactor disaster [14] suggest otherwise. These data suggest that children are at significant risk but that after the age of about 20 years, adults are, at best, at very low risk. For this reason, it is recommended that thyroid shields be worn by fluoroscopists only as a precaution, but not as a necessity.

It is recommended that fluoroscopists protect their head and neck areas with other forms of shielding. Some form of shielding placed between the exposed area of the patient and the head and neck of the fluoroscopist is highly recommended. This is easy to achieve for large patients by using appropriately mounted ceiling suspended shields. Flexible shields that are placed over the anatomy of the patient just outside the irradiated area are additionally helpful. However, use of any such shields in small children becomes problematic due to the limited working area. In these cases, thyroid shields and protective goggles are potentially reasonable alternatives to reduce exposure to the head and neck.
Legs

With the X-ray tube predominantly located under the patient, the backscatter off the posterior aspects of the patient are directed toward the floor and the shins and feet of the operator, which are not protected by the lead apron. Hair loss in fluoroscopists has been noted to occur just below the border of the lead apron [5]. Although it is not certain that this is indeed caused by the radiation, it is clear that the unprotected legs of the operator are at risk for years of cumulated dose that is not usually monitored. Because fluoroscopically guided intervention has changed the way we work with the fluoroscope, consideration to protecting the legs from long-term accumulation to high doses of radiation is strongly recommended. This can be achieved by two means:

1. Protect the legs using shields placed between the lower legs and both the X-ray tube (for leakage radiation) and the patient (for scatter)

2. Place a monitor on the trousers or top of a shoe to periodically monitor dose and use the readings to evaluate the long-term risk of radiation exposure to the legs and feet

\section{Conclusion}

Radiation-induced injury to patients and to physicians is rare. But these rare events teach us to maintain our vigilance. Recognizing that interventional work has increased our need for this vigilance, we must not be drawn into a false sense of security about the potential risks. By properly limiting dose to the patient and by properly protecting ourselves through monitoring and through shielding, our vigilance will be rewarded.

\section{References}

1. Vañó E, Arranz L, Sastre JM, et al (1998) Dosimetric and radiation protection considerations based on some cases of patient skin injuries in interventional cardiology. Br J Radiol 71:510

2. Koenig TR, Wolff D, Mettler FA, et al (2001) Skin injuries from fluoroscopically guided procedures: part I, characteristics of radiation injury. AJR 177:3-11

3. Koenig TR, Wolff D, Mettler FA, et al (2001) Skin injuries from fluoroscopically guided procedures: part II, review of 73 cases and recommendations for minimizing dose delivered to patient. AJR 177:13-20

4. Vañó E, Gonzalez L, Beneytez F, et al (1998) Lens injuries induced by occupational exposure in nonoptimized interventional radiology laboratories (review). Br J Radiol 71:728-733

5. Wagner LK (2001) Radiation injuries from fluoroscopy: a 21 st century déjà vu. In: Balter $\mathrm{S}$ (ed) Interventional fluoroscopy: physics, technology, and safety. Wiley-Liss, New York, pp 163-181

6. Food and Drug Administration (1994) Public Health Advisory: avoidance of serious $\mathrm{x}$-ray-induced skin injuries to patients during fluoroscopically-guided procedures. Center for Devices and Radiological Health, Rockville, MD 
7. Miller DL, Balter S, Wagner LK, et al (2004) Quality improvement guidelines for recording patient radiation dose in the medical record. J Vasc Interv Radiol 15:423-429

8. Hirshfeld JW, Balter S, Brinker JA, et al (2004) ACCF/AHA/ HRS/SCAI clinical competence statement on optimizing patient safety and image quality in fluoroscopically guided invasive cardiovascular procedures: a report of the American College of Cardiology/American Heart Association/American College of Physicians Task Force on Clinical Competence (ACCF/AHA/ HRS/SCAI Writing Committee to Develop a Clinical Competence Statement on Fluoroscopy). J Am Coll Cardiol 44: 2259-2282

9. Vlietstra RE, Wagner LK, Koenig T, et al (2004) Radiation burns as a severe complication of fluoroscopically guided cardiological interventions. J Interv Cardiol 17:131-142
10. Wagner LK, Eifel PJ, Geise RA (1994) Potential biological effects following high x-ray dose interventional procedures. J Vasc Interv Radiol 5:71-84

11. Wagner LK, Mulhern OR (1996) Radiation-attenuating surgical gloves: effects of scatter and secondary electron production. Radiology 200:45-48

12. Kelsey CA, Mettler FA (1990) Flexible protective gloves: the emperor's new clothes? Radiology 174:275-276

13. Holm L-E, Wiklund KE, Lundell GE, et al (1988) Thyroid cancer after diagnostic doses of iodine-131: a retrospective cohort study. J Natl Cancer Inst 80:1132

14. Ron E (1995) Thyroid cancer after exposure to external radiation: a pooled analysis of seven studies. Radiat Res 141:259-277 